\title{
ON AN EQUIVALENT CLASS OF NORMS FOR BMO
}

\author{
YONG-ZHUO CHEN and KA-SING LAU
}

(Received 28 August 1987)

Communicated by I. Raeburn

\begin{abstract}
The BMO norm of $f$ is equivalent to

$$
\sup _{(x, t) \in R_{+}^{n+1}}\left(\int|f(y)-u(x, t)|^{p} P_{t}(x-y) d y\right)^{1 / p}
$$

where $P_{t}$ is the Poisson kernel. In this note, we show that $P_{t}$ can be replaced by a nonnegative radial function $h$, which is positive in a neighbourhood of 0 , with $\int_{R^{n}} h(x) d x=1$ and $\int_{1}^{\infty} r^{n-1}(\ln r)^{p} \tilde{h}(r) d r<\infty$, where $\tilde{h}$ is the least decreasing radial majorant of $h$.

1980 Mathematics subject classification (Amer. Math. Soc.): primary 42 B 30; secondary 42 B 99.

Keywords and phrases: BMO, bounded upper means, equivalent norm, Poisson kernel.
\end{abstract}

\section{Introduction}

Let $f$ be a locally integrable function on $R^{n}$. For any sphere $S$ in $R^{n}$, let

$$
m_{S}(f)=\frac{1}{|S|} \int_{S} f(x) d x
$$

where $|S|$ denote the volume of $S$. The function $f$ is said to have Bounded Mean Oscillation (BMO) if

$$
\sup _{S} \frac{1}{|S|} \int_{S}\left|f-m_{S}(f)\right|=\|f\|_{*}<\infty .
$$

(C) 1989 Australian Mathematical Society $0263-6115 / 89 \$ A 2.00+0.00$ 
It follows from the John-Nirenberg Theorem that for each fixed $1 \leq p<\infty,\|f\|_{*}$ is equivalent to

$$
\sup _{S}\left(\frac{1}{|S|} \int_{S}\left|f-m_{S}(f)\right|^{p}\right)^{1 / p}\left(=\|f\|_{(p, *)}\right)
$$

see [3]. Let

$$
P_{t}(x)=c_{n} \frac{t}{\left(|x|^{2}+t^{2}\right)^{(n+1) / 2}}, \quad t>0,
$$

be the Poisson kernel and let $u(x, t)=\left(f * P_{t}\right)(x)$. It is also well known that the above $\|f\|_{(p, *)}$ is equivalent to

$$
\sup _{(x, t) \in R_{+}^{n+1}}\left(\int|f(y)-u(x, t)|^{p} P_{t}(x-y) d y\right)^{1 / p}
$$

where $R_{+}^{n+1}=R^{n} \times R^{+}$.

In this note we will extend the equivalence (1.2) to a larger class of kernels. Let $h$ be a nonnegative radial function on $R^{n}$ (that is, $h(x)=h(y)$ if $|x|=|y|$ ), define $h_{t}, m_{h_{t}}(f)$ by

$$
h_{t}(x)=t^{-n} h(x / t), \quad t>0 ; \quad m_{h_{t}}(f)=\int_{R^{n}} f(x) h_{t}(x) d x
$$

and the least decreasing radial majorant of $h$ by

$$
\tilde{h}(r)=\sup _{|y| \geq r} h(y), \quad r \geq 0 .
$$

The notation $\simeq$ will denote the equivalence of the norms, and $\mathscr{S}_{0}$ will denote the family of spheres centered at 0 .

THEOREM 1.1. Suppose $1 \leq p<\infty$ and that $h$ is a nonnegative radial function satisfying

(i) $\int_{R^{n}} h(x) d x=1$ and $\int_{1}^{\infty} r^{n-1}(\ln r)^{p} \tilde{h}(r) d r<\infty$.

(ii) there exists $r_{0}>0, \delta>0$ such that $h(r) \geq \delta$ for $0 \leq r \leq r_{0}$. Then

$$
\sup _{S \in \mathscr{S}_{0}}\left(\frac{1}{|S|} \int_{S}\left|f-m_{S}(f)\right|^{p}\right)^{1 / p} \simeq \sup _{t>0}\left(\int\left|f-m_{h_{t}}(f)\right|^{p} h_{t}\right)^{1 / p} .
$$

By translating $f$ and by the equivalence of the BMO norm mentioned in (1.1), Theorem 1.1 implies

COROLLARY 1.2. Let $1 \leq p<\infty$, and let $h$ be as in Theorem 1.1. Then for any locally integrable $f$ on $R^{n}$,

$$
\|f\|_{*} \simeq \sup _{(x, t)} \in R_{+}^{n+1}\left(\int\left|f(y)-m_{h_{t}}\left(f_{x}\right)\right|^{p} h_{t}(x-y) d y\right)^{1 / p} .
$$

where $f_{x}(y)=f(x-y), x, y \in R^{n}$. 
Note that Theorem 1.1 localizes (1.2) at $x=0$. The special case where $n=1$ and $h$ equals the Poisson kernel $1 /\left(\pi\left(x^{2}+1\right)\right)$ is used in [2] to study the class of functions of bounded mean oscillations with respect to 0 , that is,

$$
\left.\sup _{T>0} \frac{1}{2 T} \int_{-T}^{T} \mid f-m_{T}(f)\right]\left.\right|^{p}<\infty
$$

This class plays the same role to

$$
M^{p}=\left\{f:\|f\|=\sup _{T \geq 1}\left(\frac{1}{2 T} \int_{-T}^{T}|f|^{p}\right)^{1 / p}<\infty\right\}
$$

(functions with bounded averages) as BMO to $L^{\infty}$. Another special case is $h(x)=2 \sin ^{2} x / \pi x^{2}$, which is used in [1] and [4] to consider the integrated Fourier transformation of functions in $M^{p}$. Theorem 1.1 also extends Theorems 4.5, 4.6 in [4] where the theorems were proved in connection with the Wiener's Tauberian Theorem for limit supremum.

We remark that unlike the BMO case, the expressions in Theorem 1.1 are not equivalent for different values of $p$.

The proofs of Theorem 1.1 and the remark are given in Section 2.

\section{The proofs}

Let $\Sigma=\left\{x \in R^{n}:|x|=1\right\}$ denote the surface of the unit sphere of $R^{n},|\Sigma|$ its surface area, $\sigma$ the elements of $\Sigma$, and $d \sigma$ the elements of the surface area of $\Sigma$. Also $S_{r}$ will denote the sphere in $\mathscr{S}_{0}$ with radius $r$.

LEMMA 2.1. Let $h: R^{n} \rightarrow R^{+}, \phi: R^{1} \rightarrow R^{+}$be such that

(i) $\lim _{r \rightarrow 0^{+}} r^{n} \phi(r) \tilde{h}(r)=a$, and

(ii) $\left(r^{n} \phi(r)\right)^{\prime}$ exists a.e. and $\int \tilde{h}(r)\left(r^{n} \phi(r)\right)^{\prime} d r<\infty$.

Then there exists a constant $C$ (depending only on $\phi$ and $h$ ) such that

$$
\int_{R^{n}} F(x, T) h(x) d x \leq C, \quad \text { for all } T>0,
$$

for any measurable $F: R^{n} \times R^{+} \rightarrow R^{+}$satisfying

$$
\frac{1}{\left|S_{t}\right|} \int_{S_{t}} F(x, T) d x \leq \phi(t), \quad \text { for all } t>0, T>0
$$


PROOF. The technique is to use integration by parts. For $\alpha>0$, let $S_{\alpha} \in \mathscr{S}_{0}$, and for $T>0$, let

$$
F_{T}(r)=r^{n-1} \int_{\Sigma} F\left(r_{\sigma}, T\right) d \sigma
$$

Then

$$
\begin{aligned}
& \int_{S_{\alpha}} F(x, T) h(x) d x=\int_{0}^{\alpha} \int_{\Sigma} F\left(r_{\sigma}, T\right) h\left(r_{\sigma}\right) r^{n-1} d \sigma d r \\
& \quad \leq \int_{0}^{\alpha} \tilde{h}(r) F_{T}(r) d r=\int_{0}^{\alpha} \tilde{h}(r) d\left(\int_{0}^{r} F_{T}(t) d t\right) \\
& \quad=\tilde{h}(\alpha) \int_{0}^{\alpha} F_{T}(t) d t-\int_{0}^{\alpha}\left(\int_{0}^{r} F_{T}(t) d t\right) d \tilde{h}(r) \\
& \quad \leq \tilde{h}(\alpha)\left(\alpha^{n}|\Sigma| \phi(\alpha)\right)-\int_{0}^{\alpha}\left(r^{n}|\Sigma|\right) \phi(r) d \tilde{h}(r) \\
& \quad(\text { by }(2.1) \text { and since } \tilde{h} \text { is decreasing }) \\
& =|\Sigma|\left(a+\int_{0}^{\alpha} \tilde{h}(r)\left(r^{n} \phi(r)\right)^{\prime} d r\right) .
\end{aligned}
$$

Letting $\alpha \rightarrow \infty$, the result follows from (ii).

Let

$$
U_{p}=\left\{f \in L_{\mathrm{loc}}^{1}\left(R^{n}\right): \sup _{S \in \mathscr{S}_{0}} \frac{1}{|S|} \int_{S}\left|f-m_{S}(f)\right|^{p} \leq 1\right\} .
$$

LEMMA 2.2. For $f \in U_{p}, S \in \mathscr{S}_{0}$ and for $\alpha>0$,

$$
\left|m_{\alpha S}(f)-m_{S}(f)\right| \leq \psi(\alpha),
$$

where $\psi=\alpha^{-n / p} \chi_{[0,1]}+2^{n / p}(1+2 \ln \alpha) \chi_{(1, \infty)}$.

ProOF. If $0<\alpha \leq 1$, then

$$
\begin{aligned}
\left|m_{\alpha S}(f)-m_{S}(f)\right| & \leq\left(\frac{1}{|\alpha S|} \int_{\alpha S}\left|f-m_{s}(f)\right|^{p}\right)^{1 / p} \\
& \leq\left(\frac{1}{|\alpha S|} \int_{S}\left|f-m_{S}(f)\right|^{p}\right)^{1 / p} \leq \alpha^{-n / p}
\end{aligned}
$$


If $\alpha>1$, then there exists $k$ such that $2^{k}<\alpha \leq 2^{k+1}$ and

$$
\begin{aligned}
\mid m_{\alpha S}(f) & -m_{s}(f)|\leq| m_{\alpha S}(f)-m_{2^{k} S}(f)\left|+\sum_{j=0}^{k-1}\right| m_{2^{j+1} S}(f)-m_{2^{j} S}(f) \mid \\
\leq & \left(\frac{1}{\left|2^{k} S\right|} \int_{2^{k} S}\left|f-m_{\alpha S}(f)\right|^{p}\right)^{1 / p} \\
& +\sum_{j=0}^{k-1}\left(\frac{1}{\left|2^{j} S\right|} \int_{2^{j} S}\left|f-m_{2^{j+1} S}(f)\right|^{p}\right)^{1 / p} \\
\leq & \left(\alpha / 2^{k}\right)^{n / p}\left(\frac{1}{|\alpha S|} \int_{\alpha S}\left|f-m_{\alpha S}(f)\right|^{p}\right)^{1 / p} \\
& +2^{n / p} \sum_{j=0}^{k-1}\left(\frac{1}{\left|2^{j+1} S\right|} \int_{2^{j+1} S}\left|f-m_{2^{j+1} S}(f)\right|^{p}\right)^{1 / p} \\
\leq & 2^{n / p}\left(1+\log _{2} \alpha\right) \\
\leq & 2^{n / p}(1+2 \ln \alpha) .
\end{aligned}
$$

ProOf OF THEOREM 1.1. We first show that

$$
\sup _{T>0} \int_{R^{n}}\left|f-m_{h_{T}}(f)\right|^{p} h_{T} \leq C_{1} \sup _{S \in \mathscr{S}_{0}} \frac{1}{|S|} \int_{S}\left|f-m_{S}(f)\right|^{p}
$$

for some $C_{1}>0$. Without loss of generality we assume that $f \in U_{p}$. For $\alpha$, $T>0$ let $S_{\alpha} \in \mathscr{S}_{0}$ be the sphere of radius $\alpha$. Then

$$
\begin{aligned}
& \left(\frac{1}{\left|S_{\alpha}\right|} \int_{S_{\alpha}}\left|f(T x)-m_{S_{T}}(f)\right|^{p} d x\right)^{1 / p} \\
& \quad \leq\left(\frac{1}{\left|S_{\alpha T}\right|} \int_{S_{\alpha T}}\left|f(x)-m_{S_{\alpha T}}(f)\right|^{p} d x\right)^{1 / p}+\left|m_{S_{\alpha T}}(f)-m_{S_{T}}(f)\right| \\
& \quad \leq 1+\psi(\alpha)
\end{aligned}
$$

where $\psi(\alpha)$ is defined as in Lemma 2.2. By letting

$$
F(x, T)=\left|f(T x)-m_{S_{T}}(f)\right|^{p},
$$

we have

$$
\frac{1}{\left|S_{\alpha}\right|} \int_{S_{\alpha}} F(x, T) d x \leq(1+\psi(\alpha))^{p} \leq 2^{p}\left(1+\psi^{p}(\alpha)\right) .
$$

Let $\phi(\alpha)=2^{p}\left(1+\psi^{p}(\alpha)\right)$. Then

$$
\left(\alpha^{n} \phi(\alpha)\right)^{\prime}=\left\{\begin{array}{l}
n 2^{p} \alpha^{n-1}, \quad 0 \leq x \leq 1, \\
2^{p} \alpha^{n-1}\left[n+2^{n}(1+2 \ln \alpha)^{p-1}(n+2 n \ln \alpha+2 p)\right], \quad x>1 .
\end{array}\right.
$$

The assumption that $\int_{1}^{\infty} r^{n-1}(\ln r)^{p} \tilde{h}(r) d r<\infty$ implies that

$$
\int_{0}^{\infty}\left(\alpha^{n} \phi(\alpha)\right)^{\prime} \tilde{h}(\alpha) d \alpha<\infty
$$


Hence by Lemma 2.1,

$$
\int_{R^{n}}\left|f(T x)-m_{S_{T}}(f)\right|^{p} h(x) d x=\int_{R^{n}} F(x, T) h(x) d x \leq C
$$

for some $C>0$. Inequality (2.2) now follows from below

$$
\begin{aligned}
\left(\int_{R^{n}}\left|f(x)-m_{h_{T}}(f)\right|^{p} h_{T}(x) d x\right)^{1 / p} \\
\quad=\left(\int_{R^{n}}\left|f(T x)-m_{h_{T}}(f)\right|^{p} h(x) d x\right)^{1 / p} \\
\quad \leq\left(\int_{R^{n}}\left|f(T x)-m_{S_{T}}(f)\right|^{p} h(x) d x\right)^{1 / p}+\left|m_{h_{T}}(f)-m_{S_{T}}(f)\right| \\
\quad \leq 2\left(\int_{R^{n}}\left|f(T x)-m_{S_{T}}(f)\right|^{p} h(x) d x\right)^{1 / p} \leq 2 C .
\end{aligned}
$$

For the reverse inequality of the equivalence, we let $c=2\left(\delta\left|S_{r_{0}}\right|\right)^{-1 / p}$, and for any $T>0$, let $T^{\prime}=T r_{0}$. It follows that

$$
\begin{aligned}
\left(\frac{1}{\left|S_{T^{\prime}}\right|} \int_{S_{T^{\prime}}}\right. & \left.\left|f(x)-m_{S_{T^{\prime}}}(f)\right|^{p} d x\right)^{1 / p} \\
& \leq\left(\frac{1}{\left|S_{T^{\prime}}\right|} \int_{S_{T^{\prime}}}\left|f(x)-m_{h_{T}}(f)\right|^{p} d x\right)^{1 / p}+\left|m_{h_{T}}(f)-m_{S_{T^{\prime}}}(f)\right| \\
& \leq 2\left(\frac{1}{\left|S_{T^{\prime}}\right|} \int_{S_{T^{\prime}}}\left|f(x)-m_{h_{T}}(f)\right|^{p} d x\right)^{1 / p} \\
& \leq 2\left(\frac{1}{\left|S_{r_{0}}\right|} \int_{s_{r_{0}}}\left|f(T x)-m_{h_{T}}(f)\right|^{p} d x\right)^{1 / p} \\
& \leq c\left(\int_{S_{r_{0}}}\left|f(T x)-m_{h_{T}}(f)\right|^{p} h(x) d x\right)^{1 / p} \\
& \leq c\left(\int_{R^{n}}\left|f(T x)-m_{h_{T}}(f)\right|^{p} h(x) d x\right)^{1 / p} \\
& \leq c\left(\int_{R^{n}}\left|f(x)-m_{h_{T}}(f)\right|^{p} h_{T}(x) d x\right)^{1 / p} .
\end{aligned}
$$

To justify the remark in Section 1 , we let $1 \leq p<q<\infty$, and let

$$
A_{j}=\left\{x \in R^{1}: 2^{j} \leq|x|<2^{j}+2^{j / 2}\right\} \text {. }
$$


Define

$$
f(t)=\sum_{j=0}^{\infty} 2^{j / 2 p} \chi_{A_{j}}(t) \operatorname{sgn}(t), \quad t \in R .
$$

Since $f$ is an odd function, $m_{[-T, T]}(f)=0$, for all $T>0$. For $2^{k} \leq T<2^{k+1}$,

$$
\frac{1}{2 T} \int_{-T}^{T}\left|f-m_{[-T, T]}(f)\right|^{p} \leq \frac{1}{2^{k+1}} \sum_{j=0}^{k} \int_{A_{j}}\left|2^{j / 2 p}\right|^{p} d t \leq 2 .
$$

On the other hand, for $T=2^{k}$,

$$
\frac{1}{2 T} \int_{-T}^{T}\left|f-m_{[-T, T]}(f)\right|^{q} \geq \frac{2^{k q / 2 p}}{2^{k / 2}}
$$

which tends to $\infty$ as $h \rightarrow \infty$.

\section{References}

[1] Y. Z. Chen and K. S. Lau, 'Wiener transformation on functions with bounded means', submitted.

[2] Y. Z. Chen and K. S. Lau, 'Some new classes of Hardy spaces', J. Funct. Anal., to appear.

[3] J. B. Garnett, Bounded analytic functions (Academic Press, New York, 1981).

[4] K. S. Lau and J. Lee, 'On generalized harmonic analysis', Trans. Amer. Math. Soc. 259 (1980), 75-97.

Department of Mathematics

and Statistics

Bowling Green State University

Bowling Green, Ohio 43403

U.S.A. 